Document downloaded from:

http://hdl.handle.net/10251/120917

This paper must be cited as:

Ilisie, V.; Giménez-Alventosa, V.; Moliner Martínez, L.; Sánchez, F.; González Martínez, AJ.; Rodríguez-Álvarez, M.; Benlloch Baviera, JM. (2018). Building blocks of a multi-layer PET with time sequence photon interaction discrimination and double Compton camera. Nuclear Instruments and Methods in Physics Research Section A Accelerators Spectrometers Detectors and Associated Equipment. 895:74-83. https://doi.org/10.1016/j.nima.2018.03.076

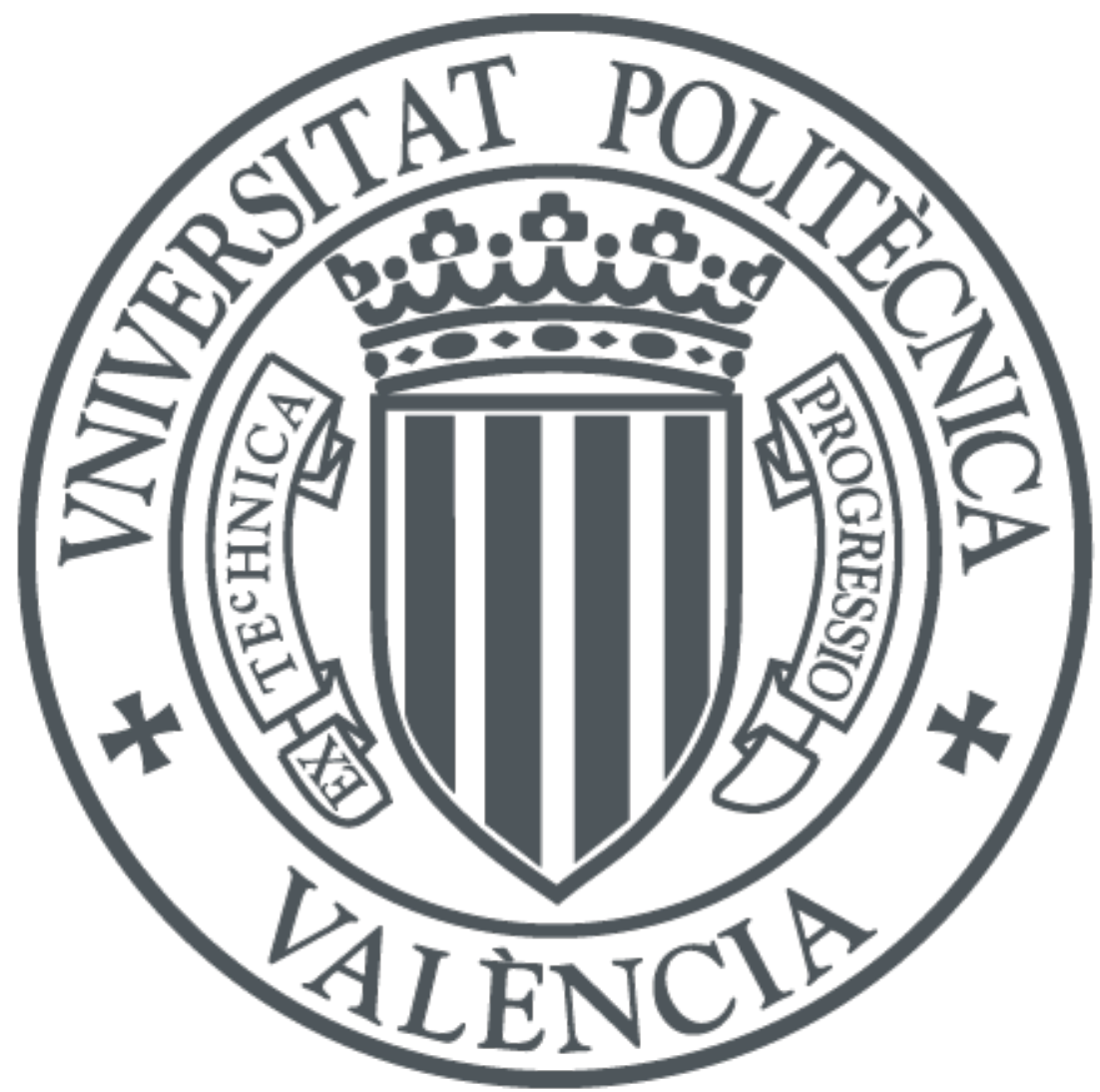

The final publication is available at

http://doi.org/10.1016/j.nima.2018.03.076

Copyright Elsevier

Additional Information 


\section{Accepted Manuscript}

Building blocks of a multi-layer PET with time sequence photon interaction discrimination and double Compton camera

V. Ilisie, V. Giménez-Alventosa, L. Moliner, F. Sánchez, A.J. González, M.J. Rodríguez-Alvarez, J.M. Benlloch

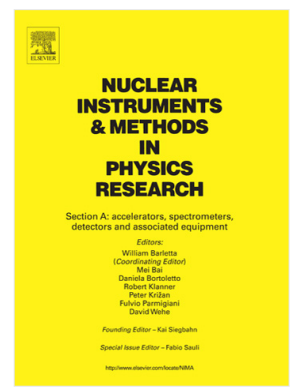

PII: $\quad$ S0168-9002(18)30443-1

DOI: $\quad$ https://doi.org/10.1016/j.nima.2018.03.076

Reference: $\quad$ NIMA 60718

To appear in: $\quad$ Nuclear Inst. and Methods in Physics Research, A

Received date: 12 January 2018

Revised date: 7 March 2018

Accepted date : 30 March 2018

Please cite this article as: V. Ilisie, V. Giménez-Alventosa, L. Moliner, F. Sánchez, A.J. González, M.J. Rodríguez-Alvarez, J.M. Benlloch, Building blocks of a multi-layer PET with time sequence photon interaction discrimination and double Compton camera, Nuclear Inst. and Methods in Physics Research, A (2018), https://doi.org/10.1016/j.nima.2018.03.076

This is a PDF file of an unedited manuscript that has been accepted for publication. As a service to our customers we are providing this early version of the manuscript. The manuscript will undergo copyediting, typesetting, and review of the resulting proof before it is published in its final form. Please note that during the production process errors may be discovered which could affect the content, and all legal disclaimers that apply to the journal pertain. 


\title{
Building blocks of a multi-layer PET with time sequence photon interaction discrimination and double Compton camera
}

V. Ilisie ${ }^{a, *}$, V. Giménez-Alventosa ${ }^{a}$, L. Moliner ${ }^{\mathrm{a}}$, F. Sánchez ${ }^{\mathrm{a}}$, A. J. González ${ }^{\mathrm{a}}$, M. J. Rodríguez-Alvarez ${ }^{\mathrm{a}}$, J. M. Benlloch ${ }^{\mathrm{a}}$

${ }^{a}$ Instituto de Instrumentación para Imagen Molecular (I3M),

Centro Mixto CSIC - Universitat Politècnica de València, Camino de Vera SN, 46022, Valencia, Spain

\begin{abstract}
Current PET detectors have a very low sensitivity, of the order of a few percent. One of the reasons is the fact that Compton interactions are rejected. If an event involves multiple Compton scattering and the total deposited energy lays within the photoelectric peak, then an energy-weighted centroid is the given output for the coordinates of the reconstructed interaction point. This introduces distortion in the final reconstructed image. The aim of our work is to prove that Compton events are a very rich source of additional information as one can improve the resolution of the detector and implicitly the final reconstructed image. This could be a real breakthrough for PET detector technology as one should be able to obtain better results with less patient radiation. Using a PET as a double Compton camera, by means of Compton cone matching i.e., Compton cones coming from the same event should be compatible, is applied to discard randoms, patient scattered events and also, to perform a correct matching among events with multiple coincidences. In order to fully benefit experimentally from Compton events using monolithic scintillators a multi-layer configuration is needed and a good time-of-flight resolution.
\end{abstract}

Keywords: PET, monolithic scintillator, Compton camera, TOF, multi-layer

\footnotetext{
*Corresponding author

Email address: victor.ilisie@i3m.upv.es (V. Ilisie)
} 


\section{Introduction}

State-of-the-art PET detectors leave plenty of room for improvement. In this work, our approach is to radically change the way Compton interactions are taken into account. The main goal is to show that being able to distinguish among the interactions from the same event i.e., with a good event tag timing resolution and with a good spatial resolution, by using a multi-layer detector configuration, can play a crucial role in improving PET resolution and sensitivity and thus, on the quality of the final reconstructed image. This could potentially lead to a reduction on the dose applied to the patient.

As a first part of this work we shortly present the most relevant details of current PET technology and focus on the special details that will be needed later in our analysis. For the second part of this work, as a theoretical exercise, we will present with an example the substantial improvement that one can obtain on the reconstructed image of a Derenzo phantom [1] in the limiting (ideal) case, in which one is able to precisely distinguish among all the interaction points within the detector. As a third part of the paper, we will present the Compton cone matching algorithm giving all the technical details, introducing a novel technique. We will also analyse the relevant sources of uncertainty i.e., detector resolution, Doppler-broadening effect, etc. and present an alternative approach for their estimation. As for the last part, we shall perform the physical characterization of the building blocks of such a Compton PET.

We defer for a forthcoming publication the simulation of a full PET system for different geometry configurations and number of layers, using all the building blocks and algorithms presented herein.

\section{Photoelectric vs. Compton}

As it is widely known, PET systems are exclusively based on the photoelectric effect and Compton interactions are purely sources of noise as described in the following. Consider a $511 \mathrm{keV} \gamma$-ray initiated within a patient's body, and for simplicity, consider that this photon does not suffer scattering within 
Table 1: Comparison between the probabilities for photoelectric, Compton and Rayleigh interactions for LYSO and $\mathrm{LaBr}_{3}$ for a $511 \mathrm{keV}$ incident photon.

\begin{tabular}{cccc}
\hline Scintillator & Photoelectric & Compton & Rayleigh \\
\hline LYSO & $45.5 \%$ & $48.8 \%$ & $5.7 \%$ \\
$\mathrm{LaBr}_{3}$ & $29.2 \%$ & $65.2 \%$ & $5.6 \%$ \\
\hline
\end{tabular}

the patient. If this photon is directly absorbed via a photoelectric effect when reaching the detector without any previous interactions (within the detector) this event will be registered. This would be the best case, for which the only sources of blurring for the coordinates of the interaction point are the intrinsic resolution of the detector and the noise introduced by the electronics. However, such cases are quite rare and one normally expects a $\gamma$-ray to previously suffer from Compton interactions (within the detector) before it is finally absorbed. This type of events will also be taken into account by the detector if the total deposited energy lays within the photoelectric peak (which will have a width associated to the detector resolution). This last case will translate into a distortion of the coordinates of the reconstructed interaction point, which implies a distorted line of response (LOR) that finally translates into a blurred reconstructed image. What is normally done in order to minimize the impact of the Compton interactions is to use materials that favour the photoelectric effect such as LYSO/LSO scintillators. In Table 1 we show a comparison between LYSO and $\mathrm{LaBr}_{3}$ for the probabilities associated to the types of interaction that can occur within the material. ${ }^{1}$ All the statistics presented in this work were extracted from Monte Carlo simulations performed independently using PENELOPE 2014 [2, 3, 4] and GATE 8.0 [5]. We always find perfect agreement within an error less than $1 \%$.

We can observe that for LYSO scintillators, the Compton and the photoelec-

\footnotetext{
${ }^{1}$ For this simulation we use a $100 \times 100 \times 20 \mathrm{~mm}^{3}$ detector block with a $511 \mathrm{keV} \gamma$-ray point source and normal incidence, in order to characterize the material intrinsically and avoid possible border effects.
} 
tric effect compete, as they have similar probabilities of interaction, meanwhile for $\mathrm{LaBr}_{3}$ the Compton effect dominates by a factor 2 . This is obviously the reason why $\mathrm{LaBr}_{3}$ or similar materials are widely used for Compton cameras $[6,7,8,9,10,11,12,13]$.

Let's now take a global look at the distribution of hits (interactions) and events (one or more hits that originate from the same incident $\gamma$-ray) that occur in a detector. In Fig. 1, for the same previous simulation, in the top-left panel we have plotted the ROOT [14] histogram of the hits as a function of the deposited energy within the detector for the LYSO block. No energy blurring has been inserted. We can observe that in the case of LYSO a considerable number of events are just photoelectric with no previous scattering (the right peak at $511 \mathrm{keV})$. On the top-right panel we show the events histogram as a function of the deposited energy (with an energy uncertainty of $10 \%$ associated to the detector resolution). By means of a rough number-of-events-per-bin counting we can observe that the four highest bins (from the photoelectric peak of the histogram of events) already sum the number of all the events from the $511 \mathrm{keV}$ photoelectric peak of the (top-left) histogram of the hits. This means that a significant number of events that lie on the photoelectric peak are the Comptonphotoelectric mixture that we have already mentioned. These noisy events are not dominant in the case of LYSO but their percentage is still, rather relevant.

Let's now take a look at the same distribution in the case of $\mathrm{LaBr}_{3}$. One can easily appreciate that the situation has changed when compared to the previous case. It can be observed that a quite small number of hits (bottom-left) are just photoelectric and that the rest (mixture of Compton plus photoelectric) dominate. Again, by performing a rough counting, already the two highest bins (from the photoelectric peak of the bottom-right histogram of events) contain the same number of events as the ones from the $511 \mathrm{keV}$ photoelectric peak from the histogram of the hits (bottom-left). This means that the a significant region of the Gaussian is pure Compton noise. Obviously this noise is much more relevant in the case of $\mathrm{LaBr}_{3}$ than in the previous case, suggesting that it is not an appropriate material for a traditional PET based on the photoelectric effect. 

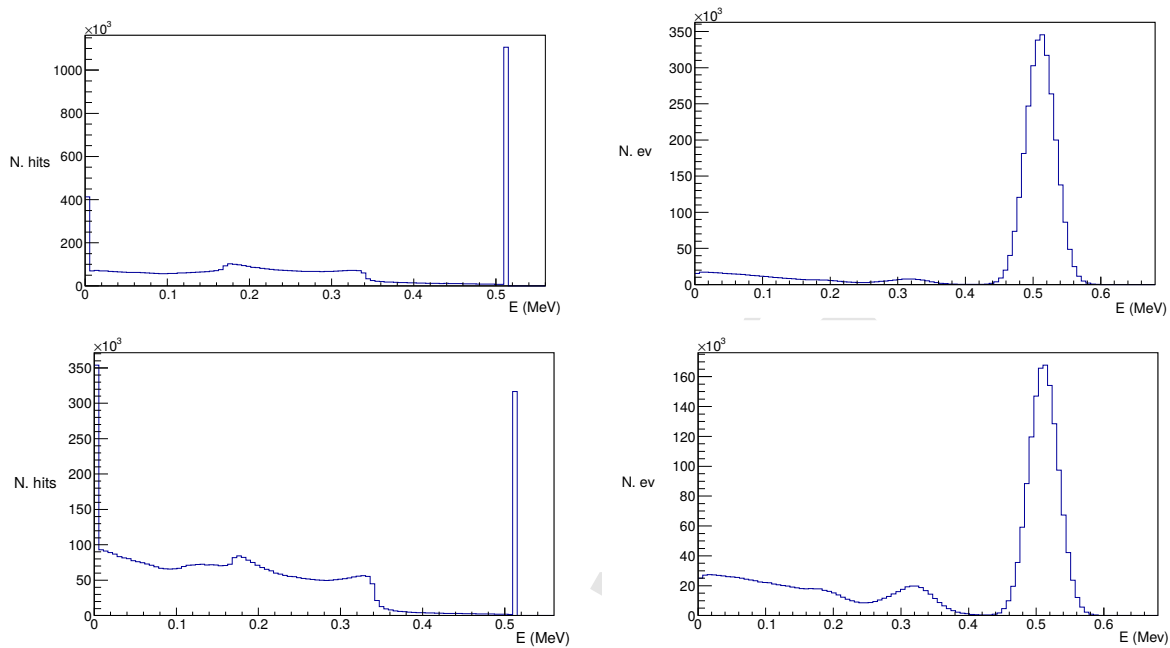

Figure 1: Histograms of the energy distribution for all individual hits for the simulated events (for a $511 \mathrm{keV}$ incident $\gamma$ ray) for LYSO (top-left) and $\mathrm{LaBr}_{3}$ (bottom-left) and histograms of the number of events as a function of the total energy deposited in the detector, with a $10 \%$ energy blurring, for LYSO (top-right) and for $\mathrm{LaBr}_{3}$ (bottom-right).

However, it is potentially an appropriate material if one intends to incorporate Compton information to the PET detector, which is the main goal of our work.

Another crucial point is the mean distance between interactions as a function of the material. If one aims to being able to distinguish among several interactions (from the same event) one must choose the appropriate material. Again, $\mathrm{LaBr}_{3}$ is more favourable from this point of view, as the mean distance between the first two consecutive interactions $d_{12}$ is greater in this case than for LYSO. In Fig. 2 we can see the distribution (in percentage) of events that suffer at least two interactions as a function of the distance $d_{12}$ (between the first two interactions) for LYSO (yellow-light) and for $\mathrm{LaBr}_{3}$ (pink/orange-dark). One can observe that, as expected, there is a much greater percentage of events that occur in the first few millimetres for LYSO than for $\mathrm{LaBr}_{3}$.

As we have already mentioned, besides the Compton scattering within the detector, there is also the possibility of Compton scattering in the patient's body. If the photon does not deposit much energy in this interaction and it 


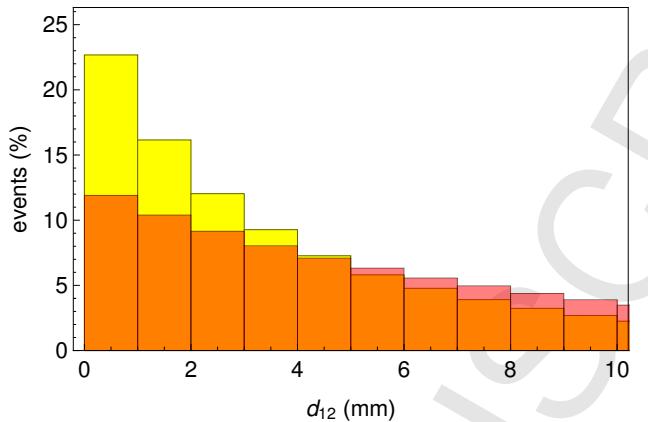

Figure 2: Distribution of events (in percentage) that suffer at least two interactions in the detector, as a function of the distance between the first two hits $d_{12}$ for LYSO (yellow-light) and $\mathrm{LaBr}_{3}$ (orange/pink-dark).

still lays on the photoelectric peak, then the detector registers this event and consequently builds the wrong LOR. There are many other sources of noise, such as random or multiple events. All these can be potentially discarded through the Compton cone matching algorithm that we shall describe is Section 4.

\section{Change of paradigm and limiting case}

Let's assume for a moment, just as a theoretical exercise, that one were able to distinguish among all the interaction points within a detector and had enough time resolution to also distinguish the time ordering of the hits. Then one would be able to recognize the coordinates of the first interaction point for each event and build the true LORs. This would obviously bring a drastic improvement to the final reconstructed image. In order to qualitatively show this improvement, we have performed the image reconstruction for a simulated Derenzo phantom in four cases.

The details of the simulation and for the image reconstruction process are given in the following. The phantom consists in tubes of $5 \mathrm{~cm}$ of length and different diameters. The specific volume activity in our case is given by

$$
A_{V}=\frac{10 \mu \mathrm{Ci}}{V_{d=2.5}}=\frac{370000 \mathrm{~Bq}}{V_{d=2.5}}
$$



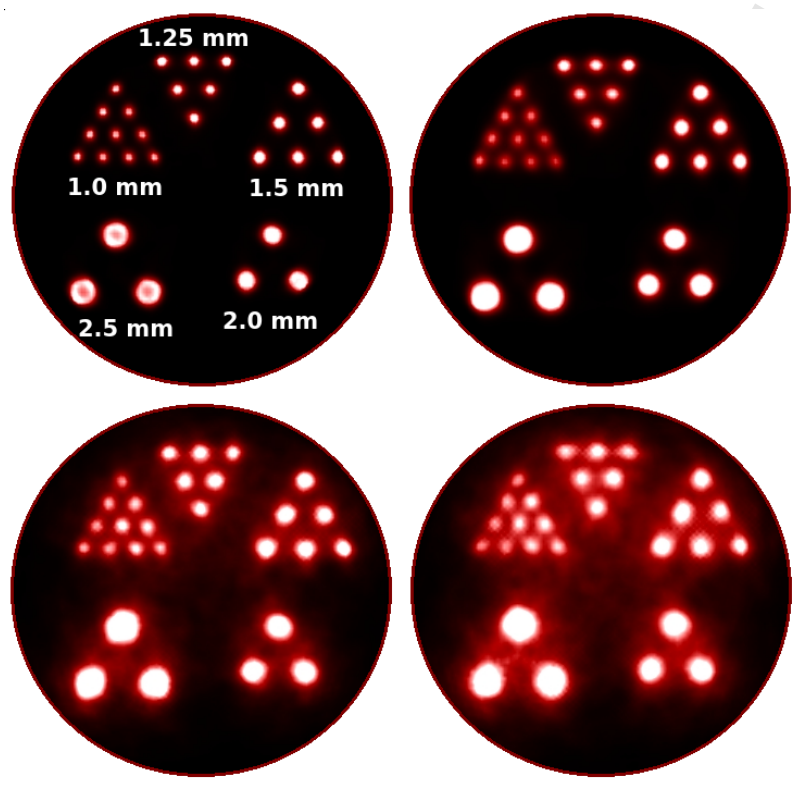

Figure 3: Image reconstruction of a GATE simulation for a Derenzo phantom for tubes of different diameters and a length of $5 \mathrm{~cm}$, for a cubical 4-block detector of dimensions $100 \times 100 \times 20 \mathrm{~mm}^{3}$, assuming that the coordinates of the first interaction points are known, with no blurring introduced (ideal image) (top-left) and with intrinsic detector blurring of 1.0 $\mathrm{mm}$ for $x$ and $y$ and $1.5 \mathrm{~mm}$ for DOI (top-right). In the bottom panel, the image reconstruction for a standard PET (using energy-averaged coordinates for events with multiple scattering) for a LYSO detector (left) and for a $\mathrm{LaBr}_{3}$ detector (right).

where $V_{d=2.5}$ is the volume of the tube of diameter $2.5 \mathrm{~mm}$. $A_{V}$ was maintained constant for all sources of the phantom. These sources were isotropic and the acquisition time was $5 \mathrm{~min}$ (with a $10 \mathrm{~ns}$ coincidence window). The detector is composed of four blocks of dimensions $100 \times 100 \times 20 \mathrm{~mm}^{3}$. The image reconstruction method was LMOS $[15,16]$ with 3 iterations and 10 subsets, $1.0 \times 1.0 \mathrm{~mm}^{2}$ virtual pixels (for the detector) and $0.25 \times 0.25 \times 0.25 \mathrm{~mm}^{3}$ voxel size.

The four cases are shown in Fig. 3. Assuming we are able to distinguish the first interaction point for each event, the ideal reconstructed image (without blurring associated to the coordinates) is shown in the top-left panel. The same image, this time introducing spatial blurring for the coordinates (associated to 
the intrinsic resolution of the detector) is shown in the top-right panel. In the bottom panels we have the image reconstruction corresponding to a standard PET detector (using the centroid method) with LYSO (left) and $\mathrm{LaBr}_{3}$ (right) scintillators.

The substantial image quality improvement can be appreciated when comparing the bottom images (that represent the images obtained with state-ofthe-art detectors) with the top-right image (which represents the maximum improvement that one can obtain over the state-of-the-art PET detectors). As this is just a simplistic theoretical exercise that is supposed to serve as a proof of concept, we have used back-to-back photons and we have only considered the image distortion introduced by uncertainty on the measurement of the coordinates. Also, we have always made the correct pairings i.e., no randoms, phantom scattering, etc. have been considered.

Having enough time and spatial resolution in order to be able to distinguish among all the interactions (originated by one event) taking place within a detector is a rather difficult task with current technology. For this reason a multi-layer approach results very appealing in this case. Further details will be given in Section 7 where we shall analyse the dominant types of events depending on the thickness of the layer.

For the moment we shall only assume that we are able to distinguish among all the interactions and that we also have knowledge of their time sequence. The purpose of doing that is to theoretically analyse all the potential information that we can extract from the Compton interactions. Details of how this can be achieved with a multi-layer configuration will be given later on.

\section{Double Compton camera and cone matching}

Using all the available information from each hit (that is, the coordinates $x, y, z$, the time of impact $t$ and the deposited energy $E$ ) in a detector, one can build a Compton cone following the equation for the Compton angle as a 

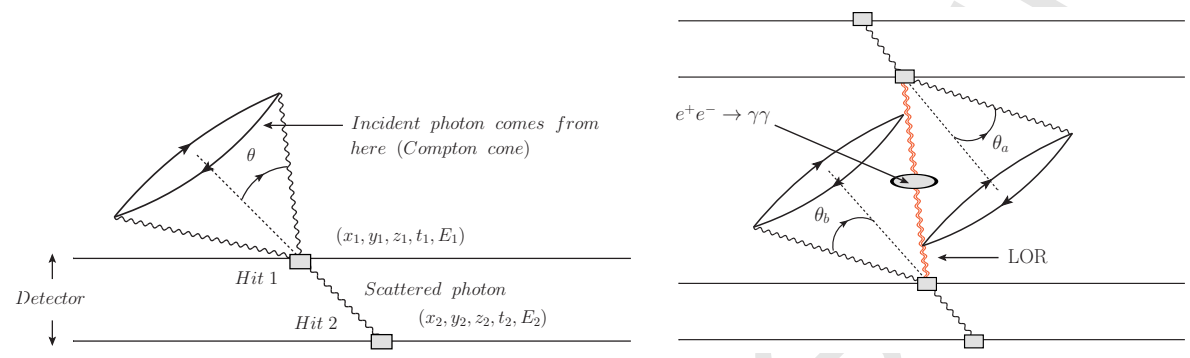

Figure 4: Reconstruction of the Compton cone using the information from the first two interactions, as in a Compton telescope (left). Two reconstructed Compton cones intersect in a LOR because they originate in the same $e^{+} e^{-}$annihilation event (right).

function of the energy

$$
\cos \theta=1-m c^{2}\left(\frac{1}{E_{2}}-\frac{1}{E_{1}+E_{2}}\right) .
$$

This is schematically shown in Fig. 4 (left) (here we have supposed for simplicity that the entire energy of the incident photon has been deposited in the two interactions i.e., $E_{1}+E_{2}=511 \mathrm{keV}$ ). This is nothing but the principle that lays beyond a Compton camera $[6,7,8]$. In a Compton camera, in order to reconstruct the image of a source one uses the intersection of many such cones. Here we propose a novel technique, where we won't only use the information from one detector, but two at the same time. This is done in order to fully benefit from the fact that we have a full PET detector and not a simple Compton camera. The philosophy of a double Compton camera that we propose is rather different and it can be described by the following principle. If two photons come from the same event, i.e., from the same electron-positron annihilation, both their Compton cones must intersect in a LOR. ${ }^{2}$ This is schematically shown in Fig. 4 (right). Thus, when multiple events are registered in a detector in the same coincidence time window, one builds all the possible Compton cones and matches the correct events by simply requiring the Compton cones to be

\footnotetext{
${ }^{2}$ This was also proposed some years ago [17], however no further study of this technique
} has been performed, to our knowledge. 

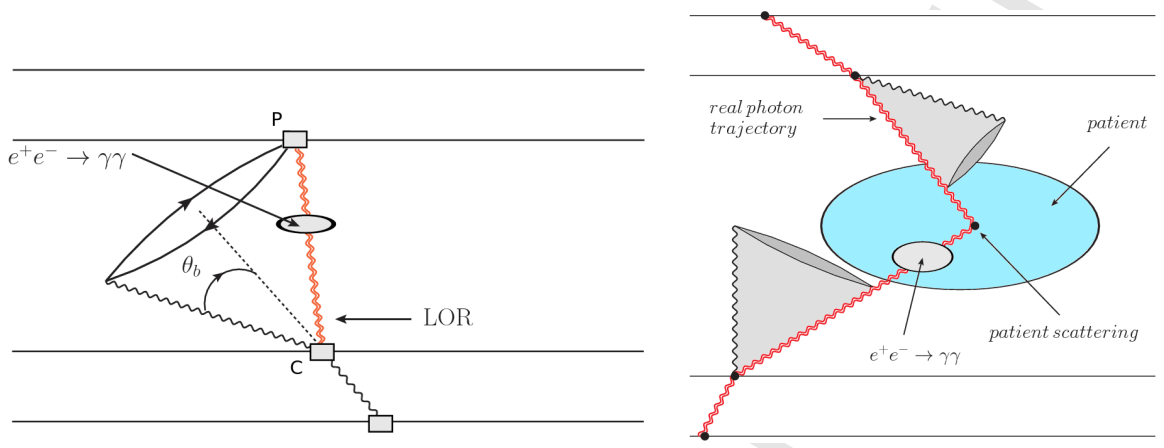

Figure 5: Compatibility of a Compton cone with a pure photoelectric event (left). Example of two reconstructed cones that do not match, for an event with scattering within the patient's body (right).

compatible (to intersect in a LOR).

In order to perform the cone matching described previously, at least two interactions (one of them Compton scattering) were required in each of the two detectors. There is also a simpler version of the matching principle. One can also match a Compton cone with a photoelectric absorption, as it is shown schematically in Fig. 5 (left). If the segment $\overline{C P}$ belongs to the surface of the Compton cone, then these two events are compatible i.e, the segment $\overline{C P}$ is a potentially true LOR.

This technique is even more powerful. Based on the same principle, by means of cone matching, one can recognize and discard events that have previously suffered scattering within the patient's body. This is schematically shown in Fig. 5 (right). Similar considerations are valid for random and multiple events. By simply checking the compatibility between each pair of cones, one in principle, is able to recognize the true matching events and build the correct LORs. In a classical PET detector one can never be sure to make the correct matching among compatible events based on the total energy information only. 


\section{Compton algorithm}

One must realize that building cones for each event is a very expensive task mathematically and also computationally and thus, difficult to implement efficiently. There is however a very simple equivalent and novel approach that we propose. Suppose we have detected 4 hits in the same coincidence time window: $A_{1}$ and $B_{1}$ in one detector and $A_{2}$ and $B_{2}$ in another one. Again, for simplicity, we shall suppose that both initial incident photons have deposited all their energy in the detectors. Using the time stamp information one can establish the time sequence of the hits. For the following calculations we shall assume (without loss of generality) that $t_{B_{1}}>t_{A_{1}}$ and $t_{B_{2}}>t_{A_{2}}$ (see Fig. 6 (a) and (b) for more details).

The next step is to construct the two geometrical angles, which we shall call $\theta_{g, 1}$ and $\theta_{g, 2}$, by using the spatial coordinates of $A_{1}, A_{2}, B_{1}$ and $B_{2}$. Assigning the coordinates as follows

$$
A_{i}\left(x_{A_{i}}, y_{A_{i}}, z_{A_{i}}\right), \quad B_{i}\left(x_{B_{i}}, y_{B_{i}}, z_{B_{i}}\right)
$$

with $i=1,2$, one can construct the following vectors

$$
\begin{aligned}
& \vec{u}_{1}=\left(x_{A_{1}}-x_{A_{2}}, y_{A_{1}}-y_{A_{2}}, z_{A_{1}}-z_{A_{2}}\right), \\
& \vec{v}_{1}=\left(x_{B_{1}}-x_{A_{1}}, y_{B_{1}}-y_{A_{1}}, z_{B_{1}}-z_{A_{1}}\right),
\end{aligned}
$$

and

$$
\vec{u}_{2}=-\vec{u}_{1}, \quad \vec{v}_{2}=\left(x_{B_{2}}-x_{A_{2}}, y_{B_{2}}-y_{A_{2}}, z_{B_{2}}-z_{A_{2}}\right) .
$$

The cosines of the two angles $\theta_{g, 1}$ and $\theta_{g, 2}$ will be simply given by the scalar product divided by the corresponding product of the moduli of the vectors

$$
\cos \theta_{g, i}=\frac{\vec{u}_{i} \cdot \vec{v}_{i}}{u_{i} v_{i}}
$$

with $i=1,2$. Assigning $E_{A_{i}}$ and $E_{B_{i}}$ (again with $i=1,2$ ) as the deposited energies in the four hits one can also calculate the cosines of the Compton angles by using (2)

$$
\cos \theta_{i}=1-m c^{2}\left(\frac{1}{E_{B_{i}}}-\frac{1}{E_{A_{i}}+E_{B_{i}}}\right) .
$$


(a)

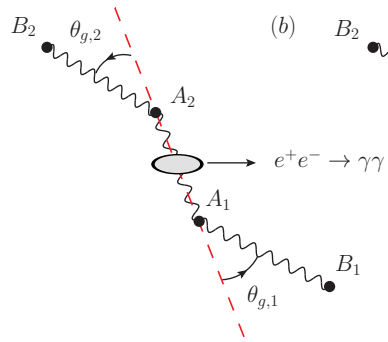

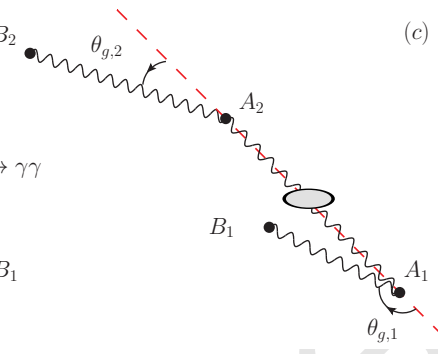

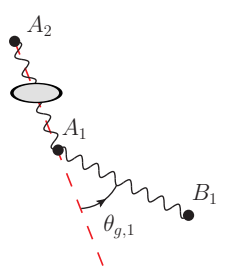

Figure 6: Schematic representation of the construction of the two geometrical angles $\theta_{g, 1}$ and $\theta_{g, 2}$ with the time ordering given by $t_{B_{2}}>t_{A_{2}}$ and the two possibilities (a) and (b) for $t_{B_{1}}>t_{A_{1}}$, when at least two interactions from the same event occur in each detector (same considerations can be made for $t_{A_{2}}$ and $t_{B_{2}}$ ). Representation of the construction of only one geometrical angle (c) for the case in which one photoelectric effect occurs in one detector and a Compton scattering in the other.

With all these ingredients and keeping in mind that the domains of both the Compton and the geometrical angles are restricted to the first two quadrants i.e., $0 \leq \theta_{g, i} \leq \pi$ and $0 \leq \theta_{i} \leq \pi$, the compatibility of the Compton cones can be resumed in the following statements in terms of angles or equivalently in terms of cosines:

(i) If $\theta_{1}=\theta_{g, 1}\left(\right.$ or $\left.\cos \theta_{1}=\cos \theta_{g, 1}\right)$ and $\theta_{2}=\theta_{g, 2}\left(\right.$ or $\left.\cos \theta_{2}=\cos \theta_{g, 2}\right)$ then the Compton cones are compatible.

We have also commented the case in which, for example $A_{2}$ is a photoelectric interaction and thus, $B_{2}$ does not exist, as in case (c) from Fig. 6. In this case, the compatibility of the Compton cone with the photoelectric event can be simply stated (in terms of angles or cosines) as

(ii) If $\theta_{1}=\theta_{g, 1}$ (or $\left.\cos \theta_{1}=\cos \theta_{g, 1}\right)$ then the Compton cone is compatible with the pure photoelectric event.

This simply means that $A_{2}$ (which is where the photoelectric effect took place) belongs to the surface of the Compton cone constructed with the information from the other detector, as shown previously in Fig. 5 (left).

The case of multiple hits in the same coincidence time window including 
randoms, singles, etc., and the complete pairing algorithm will be presented in detail in a future publication.

\section{Energy and position errors}

We have made the compatibility statements in terms of angles and in terms of cosines because for the error propagation analysis, working in terms of cosines (and not in terms of angles) will be free of divergences. All our previous formulae allow us to calculate cosines of angles (not angles) as functions of some variables i.e., $\cos \theta=f\left(x_{i}\right)$. Thus, in order to obtain the angle one needs to further apply the arccos function: $\theta=\arccos f\left(x_{i}\right)$. Let's now apply the standard error propagation formula for $\theta$. Considering that $\sigma_{x_{i}}$ are the Gaussian errors of the variables $x_{i}$ then

$$
\begin{aligned}
\sigma_{\theta}^{2} & =\sum_{j}\left(\frac{\partial \theta}{\partial x_{j}}\right)^{2} \sigma_{x_{j}}^{2}=\sum_{j}\left(\frac{\partial \arccos f\left(x_{i}\right)}{\partial x_{j}}\right)^{2} \sigma_{x_{j}}^{2} \\
& =\sum_{j}\left(\frac{\partial f\left(x_{i}\right)}{\partial x_{j}}\right)^{2} \frac{\sigma_{x_{j}}^{2}}{1-f\left(x_{i}\right)^{2}} .
\end{aligned}
$$

The function

$$
\left(1-f\left(x_{i}\right)^{2}\right)^{-1}=\left(\sin ^{2} \theta\right)^{-1}
$$

diverges when $\theta$ approaches 0 or $\pi$, which are values that do belong to our domain. When working directly with $\cos \theta$ the divergent term (9) is simply absent

$$
\sigma_{\cos \theta}^{2}=\sum_{j}\left(\frac{\partial f\left(x_{i}\right)}{\partial x_{j}}\right)^{2} \sigma_{x_{j}}^{2} .
$$

As we have concluded in the previous section, the cone matching algorithm is equivalent to comparing cosines of geometrical angles $\cos \theta_{g, i}$ and Compton angles $\cos \theta_{i}$. Each of these angles will have an associated error, one due to the uncertainty in the energy measurement and the other due to the uncertainty in the measurement of position of the interaction. Another source of error that can affect our matching algorithm is non-collinearity. So far we have assumed 
that the two emitted photons are back-to-back and this is in general not true. This source of error has been widely analysed [18] and it is known to be $\pm 0.25^{\circ}$ $\left(0.5^{\circ}\right.$ FWHM).

All these errors will be taken into account in the cosine matching algorithm in the following way. If $\theta_{g, i}$ and $\theta_{i}$ are the measured values, then if

$$
\left|\cos \theta_{g, i}-\cos \theta_{i}\right| \leqslant \epsilon_{\cos \theta}
$$

we can say that the cosines are compatible up to some confidence level given by $\epsilon_{\cos \theta}$. This last term will account for the error of the geometric angle, the Compton angle and non-collinearity. ${ }^{3}$

For the geometric angle, the errors associated to the coordinates will be given by the intrinsic spatial error of the detector. In order to be more realistic, these errors should also include noise associated to the electronics. For a multilayer detector, if the layers are different, the error will also depend on the thickness and the type of material. For the representative example analysed in Subsection 6.2 we shall use some generic values based on the latest available experimental estimations $[19,20]$. As for the energy measurement error, we have two main sources of uncertainty, the so-called Doppler-broadening effect and the error of the detector. This analysis will be performed in the following subsection.

\subsection{Compton angle error}

One important source of uncertainty is the Doppler-broadening effect. This is nothing but the effect introduced by the initial momentum of the electron. The Compton formula (2) is obtained by assuming that the electron is initially at rest. This approximation may be valid at very high energies, however in our case, the effect is rather sizeable as we shall see next. This effect can be taken

\footnotetext{
${ }^{3}$ However, as we have already mentioned at the beginning, in this work we will not present details of the full matching algorithm as it highly depends on the number of layers, and thus on the geometry of the final detector. This will be presented in a future work.
} 
into account in our Monte Carlo simulations by both PENELOPE and GATE. ${ }^{4}$

Consider an incident photon of energy $E_{\gamma}=511 \mathrm{keV}$ that deposits an amount of energy $E_{d e p}$ in a material. The energy of the outgoing photon can thus be expressed as $E_{\gamma}^{\prime}=E_{\gamma}-E_{d e p}$. The Compton formula for an electron initially at rest can then be rewritten in the form

$$
\cos \theta=1-m c^{2}\left(\frac{1}{E_{\gamma}-E_{\text {dep }}}-\frac{1}{E_{\gamma}}\right) .
$$

In Fig. 7 we can observe the prediction of this formula for a given $E_{d e p}=123$ $\mathrm{keV}$ (vertical blue-dashed line) in terms of $\cos \theta$ (left) and of $\theta$ (right). The pink-orange (dark) distribution represents the distribution due to the Dopplerbroadening effect, for the possible scattered angles for a given $E_{d e p}$ for a $\mathrm{LaBr}_{3}$ block. The yellow (light) distribution is the result of fitting the data from the previous distribution to a Gaussian. We can observe two things. First, that the distribution obviously does not follow a Gaussian distribution (it is much more peaked) and second, that it is peaked at the value given by the previous Compton formula (12). We can thus conclude that we can treat the Doppler effect as an error associated to the Compton angle given by (12) as it has already been done many times in the literature. However, by treating it as a Gaussian error we would be largely overestimating the error in many cases. For our analysis it is desirable to strictly keep the errors under control in order to precisely filter the wrong LORs by means of Compton cone matching. Our chosen approach will be not to treat this error as Gaussian but to propagate it with Monte Carlo simulation techniques as described next.

For the energy error associated to the detector, we shall suppose that it does follow a Gaussian distribution with an energy resolution of 10\% (FWHM) in one case, and $15 \%$ in a second case.

Our approach for the error propagation technique consists in the follow-

${ }^{4}$ In order to load the correct physics package that simulates the Doppler-broadening effect in GATE one has to set the physics model as PenelopeModel or LivermoreModel for the Compton interactions. The StandardModel package assumes the high energy approximation for which the initial momentum of the electron can be neglected. 

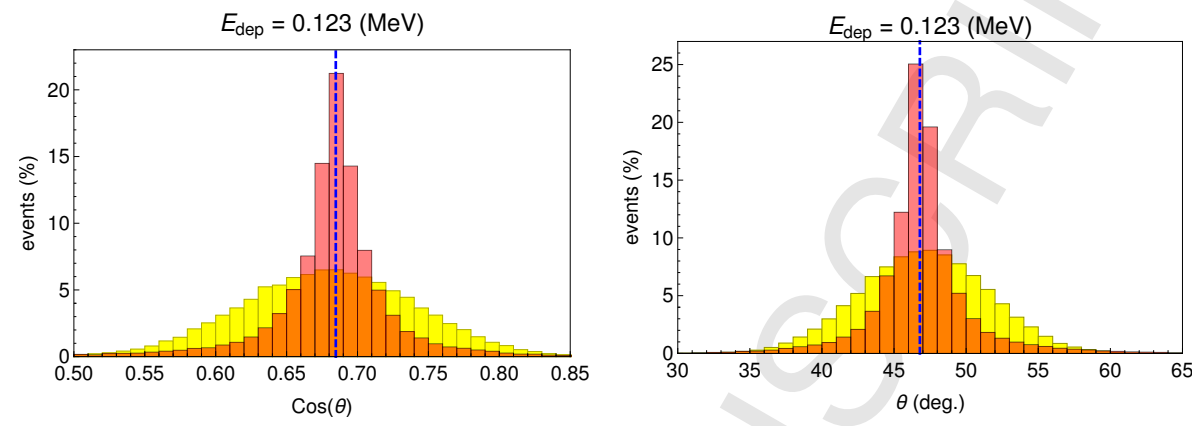

Figure 7: Distribution of $\cos \theta$ (left) and $\theta$ (right) for a given $E_{\text {dep }}=123 \mathrm{keV}$ due to the Doppler-broadening effect (pink-orange, dark) and the result of fitting the data to a Gaussian distribution (yellow, light).

ing. For each simulated event, that will obviously follow the previously shown Doppler distribution, we additionally insert a random displacement for the energy, obeying the Gaussian distribution corresponding to the uncertainty associated to the detector resolution. This way, the final distribution that we obtain accounts for both errors. The result is shown in Fig. 8 for a 10\% (left) and a $15 \%$ (right) uncertainty in the energy measurement. We can observe that in the first case the final distribution is still rather peaked and it is not well fitted by a Gaussian (which is also shown in yellow/light). In the second case as the detector error is larger, the final result is slightly better fitted by a Gaussian.

Proceeding in the same manner (using our error propagating approach) for different energies one obtains the distribution of $\cos \theta$ and its error as a function of $E_{\text {dep }}$. This is shown in Fig. 9 in orange (light) at $68.2 \%$ confidence level for an energy resolution of 10\% (FWHM). In order to obtain this error distribution we have binned the distributions of $\cos \theta$ for each given energy (that include both contributions from the Doppler effect and the energy resolution of the detector i.e., as the one shown in Fig 8) into very small bins (of $\sim 0.0001$ for $\cos \theta$ ) and we have summed the bin contributions until we reached the desired confidence level.

In Fig. 9 in blue (dark) we show the $1 \sigma$ error for $\cos \theta$ following the standard 

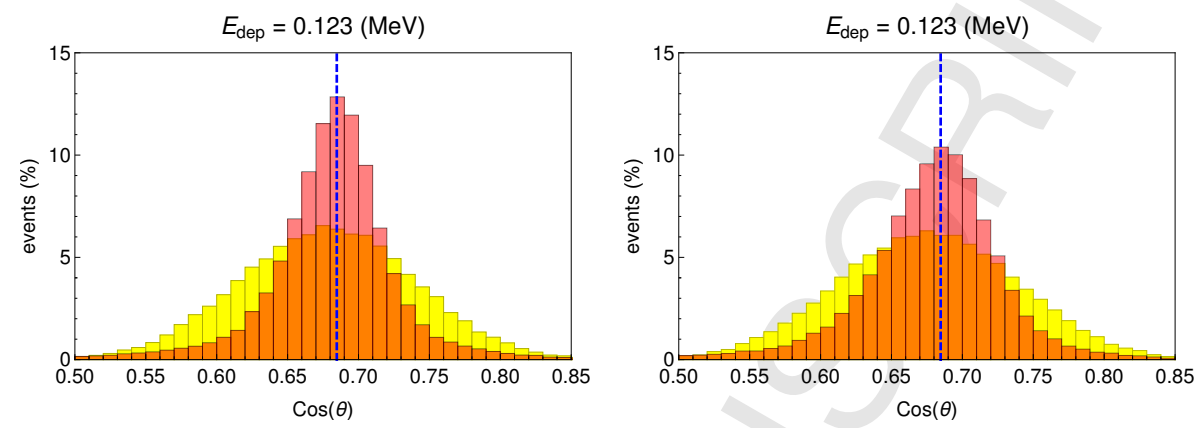

Figure 8: Error distribution of $\cos \theta$ for a given $E_{\text {dep }}=123 \mathrm{keV}$ due to both the Dopplerbroadening effect and the detector error (pink-orange, dark), and the result of fitting the data to a Gaussian distribution (yellow, light) for a 10\% (left) and 15\% (right) detector energy resolution.
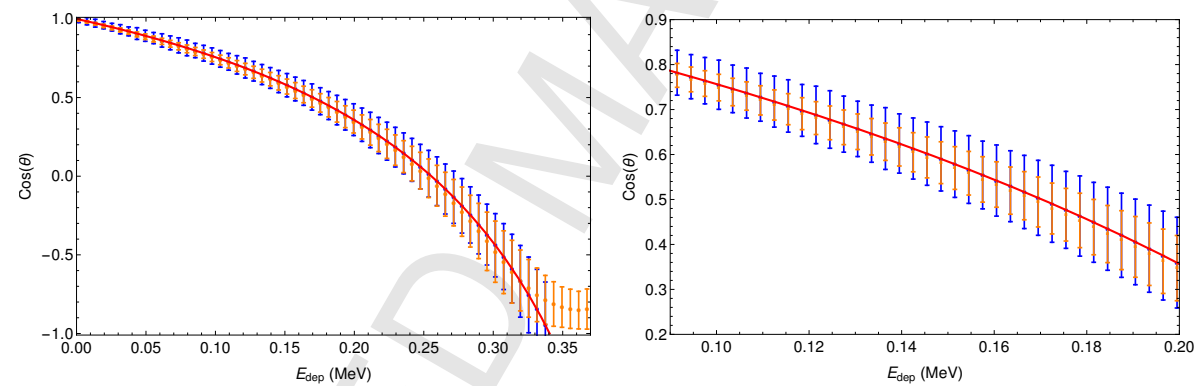

Figure 9: Left: $\cos \theta$ and its associated $68.2 \%$ confidence level error bars as a function of $E_{\text {dep }}$ using error propagation (blue, dark) and Monte Carlo techniques (orange, light). The continuous (red) line represents the function (12). Right: same as previously, zooming in one of the regions.

error propagation formula i.e.,

$$
\sigma_{\cos \theta}=\sqrt{\left(\sigma_{\cos \theta}^{\operatorname{det}}\right)^{2}+\left(\sigma_{\text {Doppler }}\right)^{2}},
$$

with

$$
\sigma_{\cos \theta}^{\text {det }}=\left|\frac{\partial \cos \theta}{\partial E_{d e p}}\right| \sigma_{E_{d e p}}=\frac{m c^{2}}{\left(E_{d e p}-E_{\gamma}\right)^{2}} \sigma_{E_{d e p}}
$$

where $\sigma_{\cos \theta}^{d e t}$ is the error of $\cos \theta$ associated to the uncertainty of the energy measurement and where $\sigma_{E_{d e p}}$ in this case is $10 \%$ FWHM (translating it into $1 \sigma$ confidence level $\left.\sigma_{E_{d e p}} \approx 0.0425 E_{d e p}\right)$. The $1 \sigma$ error associated to the Gaussian 
fit of the Doppler effect is given by $\sigma_{\text {Doppler }}$ and the final expression $\sigma_{\cos \theta}$ is given by the squared root of both contributions summed quadratically.

We can observe that the central value, using our method, is slightly shifted from the value predicted by (12). This is due to the fact that the distribution from Fig. 7 is slightly asymmetric for larger values of $E_{\text {dep }}$. We can also observe that there is a region for $E_{d e p}$ greater than $\approx 0.34 \mathrm{MeV}$, which is the maximum permitted value for $E_{d e p}$ for which $\cos \theta=-1$. This region appears due to the Doppler effect and the detector blurring that push some of the values towards that region. Thus, one can in fact experimentally measure these theoretically forbidden values of the energy and associate them to a value of $\cos \theta$ and an error. This particular region is larger than the part shown in the plot. However for our analysis we will only keep the part we have shown, as the remaining region is subject to strong statistical fluctuations due to the low number of events.

We can thus conclude that the our proposed Monte Carlo error propagation method is more realistic as it does not overestimate the errors by assuming that they follow a Gaussian distribution. Here, we have only considered $511 \mathrm{keV}$ incident photons. Similar plots can be obtained for different incident energies, that will be needed in the case of multiple hits lying in the same time coincidence window.

\subsection{Geometric angle error}

For the previous analysis we only had one parameter $E_{d e p}$. This allowed us to perform the error propagation with Monte Carlo simulations in a rather simple way. For the geometric errors however, one cannot perform such an analysis in a reasonable way for all the possible cases, performing a reasonable number of simulations or obtaining results independently of the final geometry of the detector or the layer configuration. In this case, we shall only use error propagation and assume Gaussian distributions. This, however, does not suppose a real issue as the experimentally measured values for the intrinsic spatial errors are subject to broadening effects associated to the electronics. 
Defining the auxiliary variables $x_{A_{1} A_{2}} \equiv x_{A_{1}}-x_{A_{2}}, x_{B_{1} A_{1}} \equiv x_{B_{1}}-x_{A_{1}}$ etc., by the usual error propagating method, we obtain the following result for $\sigma_{\cos \theta_{g, 1}}$ :

$$
\begin{aligned}
\sigma_{\cos \theta_{g, 1}}^{2} & =\left(\frac{x_{B_{1} A_{1}}}{u \cdot v}-\cos \theta_{g, 1} \frac{x_{A_{1} A_{2}}}{u^{2}}\right)^{2} \sigma_{x_{A_{1} A_{2}}}^{2} \\
& +\left(\frac{x_{A_{1} A_{2}}}{u \cdot v}-\cos \theta_{g, 1} \frac{x_{B_{1} A_{1}}}{v^{2}}\right)^{2} \sigma_{x_{B_{1} A_{1}}}^{2}+(x \rightarrow y, z),
\end{aligned}
$$

where the rest of the contributions to the formula are obtained, as shown schematically, by substituting $x$ by $y$ and by $z$. Similarly one can obtain the expression for $\sigma_{\cos \theta_{g, 2}}$. The expressions for $\sigma_{x_{A_{1} A_{2}}}^{2}, \sigma_{x_{B_{1} A_{1}}}^{2}$, etc., are simply given by

$$
\sigma_{x_{A_{1} A_{2}}}^{2}=\sigma_{x_{A_{1}}}^{2}+\sigma_{x_{A_{2}}}^{2}, \quad \sigma_{x_{B_{1} A_{1}}}^{2}=\sigma_{x_{B_{1}}}^{2}+\sigma_{x_{A_{1}}}^{2}, \ldots
$$

As we have already mentioned, the geometric angle error highly depends on the geometry of the detector i.e., $\cos \theta_{g, i}$ depends on the moduli of the vectors $u_{i}$ and $v_{i}$ that depend, among other things, on the inter-layer distance.

Here, for simplicity, we shall only give a representative example for the errors of $\cos \theta_{g, 1}$ for the two-layer $\mathrm{LaBr}_{3}-\mathrm{LYSO}$ configuration (Fig. 12) from Section 7. In this case we have three possible dominant combinations (case (a) from Fig. 6), and three sub-dominant ones (case (b) from Fig. 6). They are schematically shown in Fig. 10. Same analysis is valid for $\cos \theta_{g, 2}$.

We consider that the annihilation takes place within the FOV of the inner detector which is composed by the thin $\mathrm{LaBr}_{3}$ layers. ${ }^{5}$ In case (a) from Fig. 10, we consider that one of the two photons suffers a Compton interaction within the inner layer and that it is absorbed by the outer LYSO detector. As for the other photon, we consider that it interacts in another $\mathrm{LaBr}_{3}$ layer via Compton scattering or photoelectric absorption, or that it does not interact at all with this layer, but is directly absorbed by the outer LYSO detector. As for case

${ }^{5}$ The size of each $\mathrm{LaBr}_{3}$ block will be $32 \times 32 \times 3 \mathrm{~mm}^{3}$ and for the outer LYSO detector, the blocks will be of $32 \times 32 \times 20 \mathrm{~mm}^{3}$. 

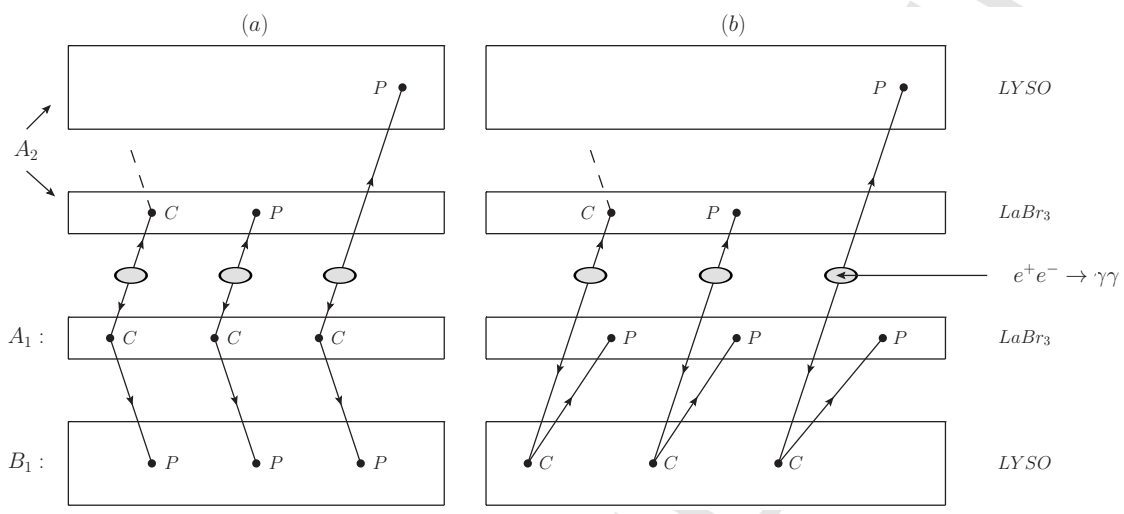

Figure 10: Schematic representation of the possible interaction configurations, for cases (a) and (b) from Fig. 6, for the interaction points $A_{1}, A_{2}$ and $B_{1}$ needed to calculate $\cos \theta_{g, 1} . P$ stands for possible multi-scattering events that end with a photoelectric absorption.

(b), it is similar to case (a), except the Compton interaction of the first photon takes place in the LYSO layer. This last configuration is subdominant.

In all cases, both (a) and (b), the dominating term in the expression for $\sigma_{\cos \theta_{g}}$ will be given by the vector whose modulus is smaller. Here we shall assign it a value of $10 \mathrm{~mm}$ (this case corresponds, for example, to an annihilation that takes place close to the corner of the inner $\mathrm{LaBr}_{3}$ cube from Fig. 12). The other modulus will have a generic value of $30 \mathrm{~mm}$, which is approximately the inter-layer distance. Spanning all possible angles, assigning an uncertainty of 1 $\mathrm{mm}$ for $x$ and $y$ and 1.5 for DOI we obtain the error distribution as a function of $\cos \theta_{g}$ from Fig 11.

\section{Multi-layer configuration}

By using current technology i.e., SiPM pixelated detectors attached to monolithic block scintillators, one is not able to distinguish among several Compton interactions with enough precision. The easiest way around in order to benefit from Compton interactions with monolithic detectors is by using a multi-layer detector. Two possible configurations are shown in Fig. 12. In order to fully exploit the potential of this multi-layer configuration several inner layers that 


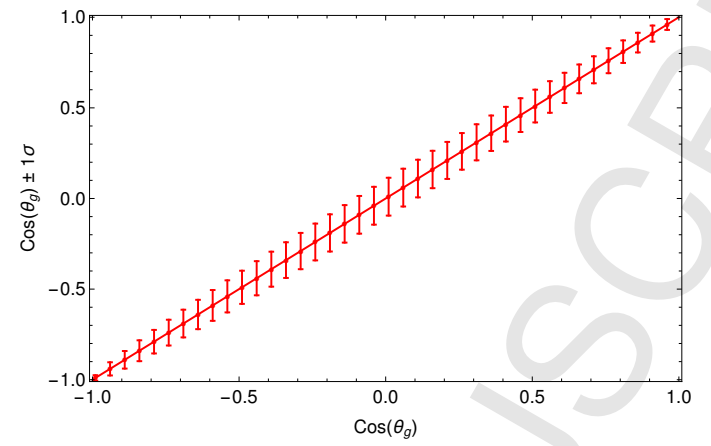

Figure 11: $\operatorname{Cos} \theta_{g} \pm 1 \sigma$ error as a function of $\cos \theta_{g}$.

favour Compton scattering should be used (such as $\mathrm{LaBr}_{3}$ ). The most outer layer should be a LYSO layer, in order to favour the photoelectric effect and thus, the total absorption of the incident photons. Also, with this configuration, if the photon passes through the inner layers (without interacting) and ends up interacting for the first time with the outer layer, the detector behaves as a traditional LYSO-based PET. The inter-layer distance should be, in principle, equal or greater than $3 \mathrm{~cm}$ in order to ensure a reasonable time-of-flight resolution $(\sim 100 \mathrm{ps})[21,22,23]$ thus, in order to be able to distinguish the time-ordering of the hits.

The choice for the thickness of the $\mathrm{LaBr}_{3}$ layer is not a trivial task and needs further analysis. For this purpose, we shall characterize the material intrinsically, independently of the geometry of the final configuration of the whole detector.

We will use a $\mathrm{LaBr}_{3}$ block of dimensions $32 \times 32 \mathrm{~mm}^{2}$ of surface ${ }^{6}$ and variable thickness $h$. Nine point sources of $511 \mathrm{keV} \gamma$-rays are placed uniformly at $3 \mathrm{~mm}$ from the detector surface, as shown in Fig. 13. The photon emission will be isotropic in order to cover for all possible incident angles. We will divide the possible interactions of this first $\mathrm{LaBr}_{3}$ layer with a $511 \mathrm{keV}$ incident photon

\footnotetext{
${ }^{6}$ Which is the size of the standard digital Philips SiPM detectors [24] that we will use for our experimental setup.
} 

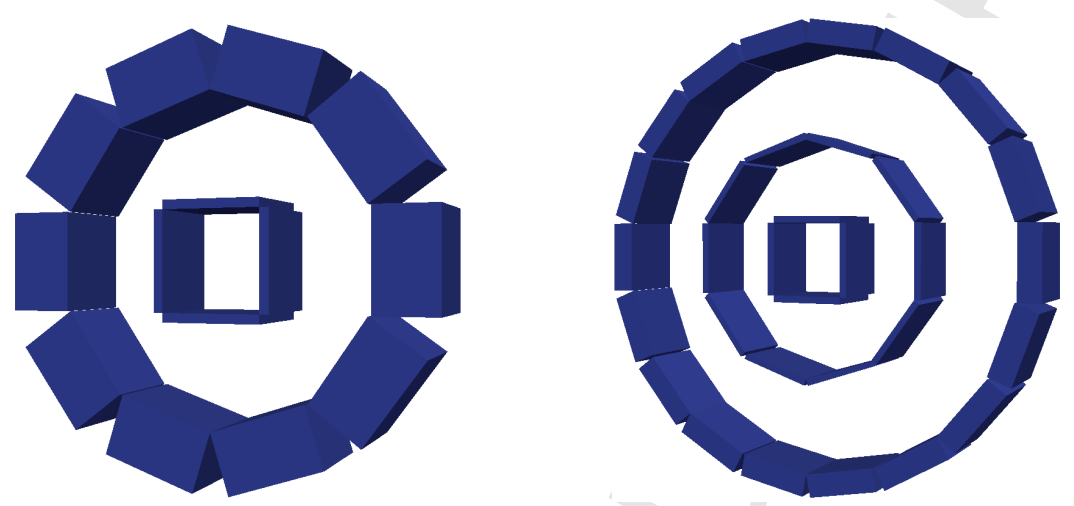

Figure 12: Representation of two possible multi-layer configurations for a PET detector. The thin inner layers are composed by $\mathrm{LaBr}_{3}$ blocks of dimensions of of $32 \times 32 \times 3 \mathrm{~mm}^{3}$, and the thick (most outer) layers are composed by LYSO blocks of dimensions $32 \times 32 \times 20 \mathrm{~mm}^{3}$. The number of blocks per layer is given by 4-10 (left) and 4-10-18 (right). The distance in between two consecutive layers is $\sim 30 \mathrm{~mm}$.

into the categories shown in Fig. 14. The possibilities are:

(a) one Compton interaction and escapes the scintillator in the forward direction (our definition of forward for this particular case is given by all the points with $z<0$ as shown in Fig 13.)

(b) one Compton interaction and escapes backward

(c) a direct photoelectric absorption

(d) two Compton interactions and escapes forward

(e) two Compton interactions and escapes backward

(f) one Compton interaction and a photoelectric effect

(g) two Compton interactions and a photoelectric effect.

Any other possibility can be safely neglected as its probability will be extremely low.

Thus, the thickness of the layer plays a key role, as it has to be as thick as possible in order to ensure a high rate of interactions with this first detector 


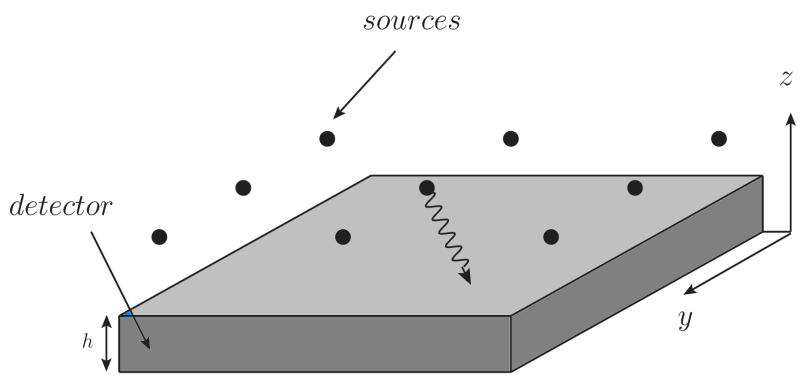

forwardregion

Figure 13: $\mathrm{A} \mathrm{LaBr}_{3}$ detector formed by a $32 \times 32 \mathrm{~mm}^{2}$ block with variable thickness $h$ and 9 point sources uniformly distributed. The region considered as forward is the region given by the negative $z$ region, where other detector layers can be placed.

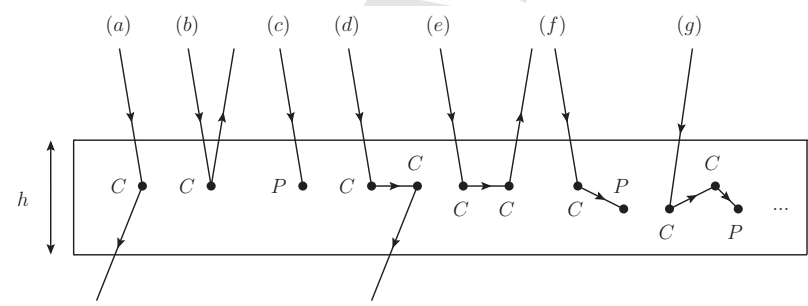

Figure 14: Categories for the possible interactions with a $\mathrm{LaBr}_{3}$ layer for a $511 \mathrm{keV}$ incident photon, where $\mathrm{C}$ stands for Compton, $\mathrm{P}$ stands for photoelectric and $h$ for the thickness of the block. The trajectory of the photon is schematically given by the arrow. If the arrow of the escaping photon points downwards it means that the photons escapes forward, whereas if it points upwards means that it escapes backward.

and, it has to be thin enough in order to obtain a dominant percentage of events of the type (a) with a small rate of events of the type (f) and (g) (events that will introduce noise). It also has to be thin enough to ensure a good spatial resolution. Here we shall analyse three possible values for $h$ : 3,4 and $5 \mathrm{~mm}$.

The results for the obtained percentages are shown in Table 2. They are calculated as follows. Out of the total number of incident photons (on the surface of the detector) that we shall denote $N_{t o t}$, the number of events that interact at least once with the layer will be represented by $N_{L_{1}}$. Thus, out of $N_{\text {tot }}$, the percentage of the events that interact at least once with the layer will 
Table 2: Percentages for the interactions with the $\operatorname{LaBr}_{3}$ layer as functions of $h(m m)$.

\begin{tabular}{cccccccccc}
\hline $\mathrm{h}$ & $L_{1}$ & $A$ & $B$ & $C$ & $D$ & $E$ & $F$ & $G$ & $L_{1, A}$ \\
\hline 3.0 & 21.2 & 41.5 & 23.4 & 14.3 & 4.2 & 3.6 & 8.6 & 2.7 & 8.8 \\
4.0 & 26.4 & 39.5 & 22.0 & 14.3 & 4.6 & 3.9 & 9.9 & 3.5 & 10.4 \\
5.0 & 30.9 & 37.9 & 20.7 & 14.3 & 5.0 & 4.0 & 11.0 & 4.4 & 11.7 \\
\hline
\end{tabular}

be given by

$$
L_{1}=\left(N_{L_{1}} / N_{t o t}\right) \cdot 100
$$

The following percentages are calculated with respect to $N_{L_{1}}$ and the categories are: $(A)$ percentage of events of the type (a) from Fig. 14 (meaning that if $N_{A}$ is the number of events of the type (a) then $\left.A=\left(N_{A} / N_{L_{1}}\right) \cdot 100\right),(B)$ percentage of events of the type (b), and so on.

The last category is given by $L_{1, A}$ which is the percentage of events of the type (a) out of $N_{t o t}$ i.e., if $N_{A}$ is the number of events of the type $A$ then

$$
L_{1, A}=\frac{N_{A}}{N_{t o t}} \cdot 100=\frac{A \cdot L_{1}}{100} .
$$

This percentage is the one that we aim to improve by increasing the thickness $h$ of the $\mathrm{LaBr}_{3}$ layer.

We can observe that even if A decreases with $h$ (obviously because the probability to suffer multi-scattering grows with $h), L_{1, A}$ increases. This is due to the fact that $L_{1}$ dominates over $\mathrm{A}$ in (18), as the number of events that interact with the layer $N_{L_{1}}$ grows faster than the decrease rate of $A$. Events of the type (c), which are photoelectric effects with no previous scattering are also useful, as they do not introduce any additional blurring. Events of the type (b) can be useful in the case of multi $\mathrm{LaBr}_{3}$ layers if it does not occur in the first layer. Finally (d) and (e) are events that can potentially be discarded through the cone matching algorithm. 


\section{Conclusions}

We have presented the physical characterization of a future PET that will incorporate the Compton effect. We have shown that Compton interactions can be a rich source of information that can help match the true coincidences and discard random, scattered and multiple events, thus building the true LORs. We have demonstrated that the matching algorithm, that was first thought in terms of cone matching, can be treated equivalently in a simple form in terms of geometric and Compton angles. We have also presented a rigorous error treatment both with Monte Carlo techniques and with standard error propagation. In a future publication we will analyse possible geometries, simulate a full PET detector and apply the Compton algorithm presented herein, in order to quantify the potential improvement over state-of-the-art PET detectors.

The physical development of this new PET apparatus is currently an undergoing process in our group. Based on the results of the simulations, the most suitable geometry will soon be chosen. For now, special efforts are being made in obtaining a good spatial and TOF resolution for $\mathrm{LaBr}_{3}$ moduli of different thickness and different inter-layer distances. The final goal of this project is to perform tests with small animals as soon as the final geometry is optimized.

\section{Acknowledgements}

This project has received funding from the European Research Council (ERC) under the European Unions Horizon 2020 research and innovation programme (grant agreement No 695536). This work was supported in part by the Spanish Government grants TEC2016-79884-C2 and RTC-2016-5186-1.

\section{References}

[1] S. Derenzo, T. Budinger, J. Cahoon, R. Huesman, H. Jackson, High resolution computed tomography of positron emitters, Nuclear Science, IEEE T Nucl Sci. 24 (1977) 544-558. 
[2] F. Salvat, PENELOPE-2011 A code system for monte carlo simulation of electron and photon transport, OECD Nuclear Energy Agengy.

[3] J. Sempau, A. Badal, L. Brualla, A PENELOPE based system for the automated Monte Carlo simulation of clinacs and voxelized geometries, application to far from axis fields, Med. Phys. 38 (11) (2011) 5887-5895. doi:10.1118/1.3643029.

[4] J. Sempau, J. M. Fernández-Varea, E. Acosta, F. Salvat, Experimental benchmarks of the Monte Carlo code PENELOPE, Nuclear Inst. and Methods in Physics Research B 207 (2) (2003) 107-123.

[5] S. Jan, et al., GATE: a simulation toolkit for PET and SPECT, Phys. Med. Biol. 49 (19) (2004) 4543-4561. doi:10.1088/0031-9155/49/19/007.

[6] R. W. Todd, J. M. Nightingale, D. B. Everett, A proposed gamma camera, Nature 251 (1974) 132-134. doi:10.1038/251132a0.

[7] D. B. Everett, J. S. Fleming, R. W. Todd, J. M. Nightingale, Gammaradiation imaging system based on the Compton effect, IEEE Proc. 124 (11) (1977) 995-1000. doi:10.1049/piee.1977.0203.

[8] V. Schoenfelder, et al., Instrument description and performance of the imaging gamma-ray telescope COMPTEL aboard the compton gamma-ray observatory, Astroph. J. Suppl. Series 86 (1993) 657-692.

[9] E. V. D. van Loef, et al., Scintillation properties of LaBr3:Ce3+ crystals: fast, efficient and high-energy-resolution scintillatiors, Nuclear Inst. and Methods A 486 (1-2) (2002) 254-258. doi:10.1016/S0168-9002(02) 00712-X.

[10] Y. Feng, R. S. Detwiler, J. E. Baciak, W. Kernan, Optimized geometry and limitations of Compton cameras with LaBr3, 2007 IEEE Nuclear Science Symposium Conference Record 2 (2007) 1227-1233. doi :10.1109/NSSMIC. 2007.4437226. 
[11] J. Barrio, A. Etxebeste, L. Granado, E. Muñoz, G. Oliver, A. Ros, J. Roser, C. Solaz, G. Llosá, Performance improvement tests of MACACO: A Compton telescope based on continuous crystals and SiPMs, Nuclear Inst. and Methods in Physics Research A,doi:10.1016/j.nima.2017.10.033.

[12] E. Muñoz, J. Barrio, A. Etxebeste, O. P. Garcia, C. Lacasta, G. Oliver, C. Solaz, G. Llosá, Performance evaluation of MACACO: a multilayer Compton camera, Phys. Med. Biol. 62 (18) (2017) 7321-7341. doi: $10.1088 / 1361-6560 /$ aa8070.

[13] F. Quarati, A. J. J. Bos, S. Brandenburg, C. Dathy, P. Dorenbos, S. Kraft, et al., X-ray and gamma-ray response of a 2" x 2" LaBr3:Ce scintillation detector, Nuclear Inst. Methods Physics Research A 574 (2007) 115-120.

[14] R. Brun, F. Rademarkers, ROOT - An object oriented data analysis framework, Proceedings AIHENP'96 Workshop, Nuclear Inst. and Methods in Physics Research A 389 (1997) 81-86.

[15] H. M. Hudson, R. S. Larkin, Accelerated image reconstruction using ordered subsets of projection data, IEEE Trans. Med. Imaging 13 (4) (1994) 601-609. doi:10.1109/42.363108.

[16] L. Moliner, C. Correcher, A. Gonzalez, P. Conde, L. Hernandez, A. Orero, M. J. Rodriguez, F. Sanchez, A. Soriano, L. F. Vidal, J. M. Benlloch, Implementation and analysis of list mode algorithm using tubes of response on a dedicated brain and breast pet, Nuclear Inst. and Methods in Physics Research A 702 (2013) 129-132. doi:10.1016/j.nima.2012.08.029.

[17] C. S. Levin, Promising new photon detection concepts for high-resolution clinical and preclinical pet, J Nucl Med. 53 (2) (2012) 167-170.

[18] T. F. Budinger, PET instrumentation: What are the limits?, Seminars in Nuclear Medicine 28 (3) (1998) 247-267. doi:10.1016/S0001-2998(98) $80030-5$. 
[19] G. Llosá, et al., Second LaBr3 Compton telescope prototype, 3rd International Conference on Advancements in Nuclear Instrumentation, Measurement Methods and their Applications (ANIMMA) (2013) 1-4, doi: 10.1109/ANIMMA. 2013.6727965.

[20] A. González-Montoro, A. Aguilar, G. Cañizares, P. Conde, L. Hernández, L. F. Vidal, M. Galasso, A. Fabbri, F. Sánchez, J. M. Benlloch, A. J. González, Performance study of a large monolithic LYSO PET detector with accurate photon DOI using retroreflector layers, IEEE Transactions on Radiation and Plasma Medical Sciences 1 (3) (2017) 229-237.

[21] V. N. Nemallapudi, S. Gundacker, P. Lecoq, E. Auffray, A. Ferri, A. Gola, C. Piemonte, Sub-100 ps coincidence time resolution for positron emission tomography with LSO:Ce codoped with Ca, Phys. Med. Biol. 60 (12) (2015) 4635-49.

[22] P. Lecoq, Pushing the limits in time-of-flight PET imaging, IEEE Transactions on Radiation and Plasma Medical Sciences 1 (6) (2017) 473-485. doi : 10.1109/TRPMS. 2017.2756674.

[23] S. Gundacker, E. Auffray, B. Frisch, P. Jarron, A. Knapitsch, T. Meyer, M. Pizzichemi, P. Lecoq, Time of flight positron emission tomography towards 100ps resolution with L(Y)SO: an experimental and theoretical analysis, Journal of instrumentation 8 (P07014) (2013) 1-29. doi: $10.1088 / 1748-0221 / 8 / 07 /$ P07014.

[24] R. Schulze, PDPC-TEK user manual, Philips digital photon counting (2013), http://www.digitalphotoncounting.com. 